\title{
Biological and biomedical implications of the co-evolution of pathogens and their hosts
}

\author{
Mark E.J. Woolhouse ${ }^{1}$, Joanne P. Webster ${ }^{2}$, Esteban Domingo ${ }^{3}$, Brian Charlesworth ${ }^{4}$ \\ \& Bruce R. Levin 5
}

\begin{abstract}
Co-evolution between host and pathogen is, in principle, a powerful determinant of the biology and genetics of infection and disease. Yet co-evolution has proven difficult to demonstrate rigorously in practice, and co-evolutionary thinking is only just beginning to inform medical or veterinary research in any meaningful way, even though it can have a major influence on how genetic variation in biomedically important traits is interpreted. Improving our understanding of the biomedical significance of co-evolution will require changing the way in which we look for it, complementing the phenomenological approach traditionally favored by evolutionary biologists with the exploitation of the extensive data becoming available on the molecular biology and molecular genetics of host-pathogen interactions.
\end{abstract}

That pathogens and hosts have evolutionary effects on one another and that these effects might be reciprocal-that is, pathogens and hosts co-evolve-are attractive, plausible and powerful ideas whose implications for the medical and veterinary sciences are only just beginning to be recognized ${ }^{1}$. An important instance concerns the genetics of susceptibility to and pathogenicity of infectious diseases. At one level, co-evolution is of interest simply as a means of maintaining polymorphisms at relevant loci ${ }^{2,3}$. But beyond this, whereas studies of susceptibility and pathogenicity generally take a static view of the underlying population genetics, co-evolution is a dynamic process: if host and pathogen co-evolve, then a 'good' gene in one time and place may be a 'bad' gene in another time and place. Failure to recognize the dynamic nature of the interaction could result in misinterpretation of its genetic basis.

Whereas there is abundant evidence consistent with co-evolution from studies of pathogens infecting invertebrates, plants or bacteria $^{3-7}$, there is less concerning pathogens of humans and other long-lived, vertebrate hosts. Although this has been taken by some to indicate that co-evolution may not be important in such hosts ${ }^{8-10}$, an alternative view is that traditional approaches to looking for co-evolution are too limited, especially when it takes place against a complex and dynamic background of constraints and trade-offs in both host and pathogen. In this respect, biomedical science may have as much to offer evolutionary biology as vice versa; rapidly accumulating data on molecular biological and molecular genetic aspects of infectious diseases are providing new opportunities for studying host-pathogen co-evolution.
In this review, we assess the evidence for co-evolution in host-pathogen systems. We then explore the complexities of studying co-evolution in the 'real world', where there are many different constraints on the potential for pathogen and host to co-evolve. We proceed to consider the largely untapped opportunity for studying co-evolution at the mechanistic as well as the phenomenological level. Finally, we offer some suggestions for future directions for studies of co-evolution and its biomedical significance.

\section{Nature of co-evolution}

Definition. We define co-evolution as the process of reciprocal, adaptive genetic change in two or more species (Fig. 1, Box 1). This simply means that changes in gene frequencies as a result of selection acting on one population create selection for changes in gene frequencies in the other population(s), although the kinds of population genetic processes that result can be different, as we detail below. Co-evolution can occur between any interacting populations: prey and predator, plant and herbivore, competitors or mutualists, but it is expected to be particularly important in host-pathogen systems because of the intimate nature of the association and the strong selective pressures that each can exert on the other ${ }^{4}$.

Examples of reciprocal traits potentially involved in co-evolution include pathogen infectivity and host resistance; host-seeking and pathogen-avoidance behaviors; and the ability of the host to clear an infection versus the ability of the pathogen to evade or suppress host defenses. In each of these pairings, the effects on the fitness of the host and pathogen act in opposite directions: what is

${ }^{1}$ Centre for Infectious Diseases, College of Medicine and Veterinary Medicine, University of Edinburgh, Easter Bush, Roslin, Midlothian EH25 9RG, UK. ${ }^{2}$ Peter Medawar Building for Pathogen Research, University of Oxford and Department of Zoology, Oxford, UK. ${ }^{3}$ Centro de Biologia Molecular, Universidad Autonoma de Madrid, Cantoblanco, Madrid, Spain. Institute of Cell, Animal and Population Biology, King's Buildings, Edinburgh, UK. ${ }^{5}$ Department of Biology, Emory University, Atlanta, Georgia, USA. Correspondence should be addressed to M.E.J.W. (e-mail: mark.woolhouse@ed.ac.uk). 


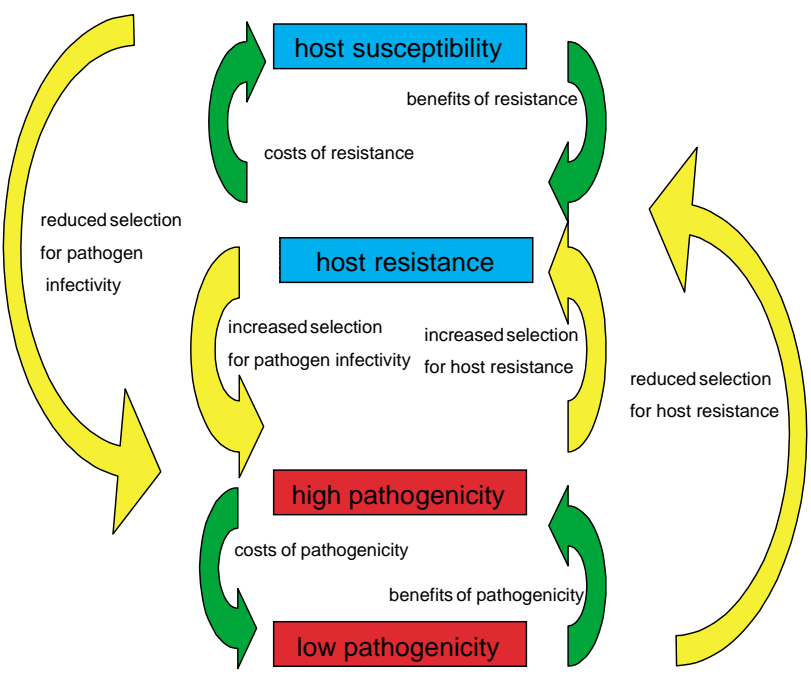

good for the pathogen is bad for the host and vice versa. There is a more ambiguous, though extremely important, relationship between the pathogen- and host-related components of virulence. Here, we take virulent to mean harmful but not necessarily highly transmissible, and note that virulence is determined by the host response to infection as well as by the biology of the pathogen itself. By definition, virulence is associated with decreased host fitness, but it may be associated with either decreased or increased pathogen fitness ${ }^{11,12}$ or, conceivably, it may not be of selective significance to the pathogen at all ${ }^{13}$.

Theory of co-evolution. Theoretical studies of co-evolution date back over 40 years ${ }^{14,15}$; examples include 'gene-for-gene', 'matching allele' and 'matching genotype' models ${ }^{16-22}$. The key feature
Fig. 1 Schematic representation of co-evolution, emphasizing reciprocity in that changes in allele frequencies due to selection in one species impose selection resulting in changes in allele frequencies in the other. Green arrows indicate intra-specific selection; yellow arrows indicate inter-specific selection.

of these models is that the outcome of the host-pathogen interaction depends on the combination of host and pathogen genotypes involved. Other features vary: whether the genome is haploid or diploid; the number of alleles involved and whether there is pleiotropy (especially fitness costs independent of the host-pathogen interaction); dominance relationships between alleles; the number of loci involved and whether there is epistasis; whether reproduction is sexual or asexual; whether mating is random or assortative; and the relative generation times of host and pathogen and whether generations are discrete or continuous. The models predict that co-evolution can occur under a wide range of conditions, but that many different outcomes are possible: stable polymorphisms; dynamic polymorphisms with cyclic or chaotic fluctuations in allele frequencies of varying amplitude; unstable cycles leading to fixation; or selective sweeps of favorable alleles to fixation. Here, we focus on two kinds of changes in allele frequencies, selective sweeps and dynamic polymorphisms, both involving reciprocal changes in host and pathogen.

Selective sweeps occur when new alleles appear, by mutation or migration, and eventually become fixed in the population. These result in transient polymorphisms, although the process of fixation may be slow (Fig. 2a). Dynamic polymorphisms involve fluctuations in allele frequencies caused by selection and are inherently persistent, although fixation can occur as a result of genetic drift (Fig. $2 b$ ). We regard Fig. $2 a$ as representing an 'arms race' (accumulated 'improvements' in both populations) and Fig. $2 b$ as representing 'Red Queen' dynamics

\section{Box $1 \cdot$ Glossary}

\begin{tabular}{|c|c|}
\hline Term & Definition \\
\hline co-evolution & $\begin{array}{l}\text { Reciprocal, adaptive genetic changes between interacting species. Co-evolution can be studied in terms of } \\
\text { paired phenotypic traits, such as resistance and infectivity, in terms of interacting host and pathogen } \\
\text { molecules or in terms of genes or nucleotide sequences directly. }\end{array}$ \\
\hline gene-for-gene & $\begin{array}{l}\text { Co-evolution involving a single locus in the genomes of each of two interacting populations. The outcome of } \\
\text { the interaction depends on the combination of alleles at these loci. }\end{array}$ \\
\hline resistance & $\begin{array}{l}\text { Genetic, biochemical or physiological characteristics of the host that inhibit pathogen establishment, survival or } \\
\text { development. Resistance may be quantitative, so that hosts may be infected but resistant ones are harmed less, } \\
\text { or qualitative, so that host infection is prevented. The majority of studies of host-pathogen co-evolution regard } \\
\text { resistance as the latter. }\end{array}$ \\
\hline infectivity & $\begin{array}{l}\text { Genetic, biochemical or physiological characteristics of the pathogen that determine its ability to infect the } \\
\text { host. High infectivity does not necessarily mean that symptoms of disease (pathology) appear more quickly, nor } \\
\text { that the illness is more severe (virulence). }\end{array}$ \\
\hline virulence & $\begin{array}{l}\text { The direct or indirect reduction in host fitness attributable to pathogen infection, often measured as pathogen- } \\
\text { induced host mortality. (Conflicting definitions exist, such as that of the infective capacity of pathogens (here } \\
\text { termed "infectivity") within plant-pathogen systems.) }\end{array}$ \\
\hline compatibility & $\begin{array}{l}\text { The ability of a given pathogen to infect a given host, as a consequence of the combined genetic, } \\
\text { biochemical or physiological characteristics that determine infectivity and resistance. }\end{array}$ \\
\hline trade-off & $\begin{array}{l}\text { An unavoidable genetic constraint/negative correlation in which investment in one trait compromises } \\
\text { investment in another. }\end{array}$ \\
\hline local adaptation & Sympatric combinations (here, of host and pathogen) are more compatible than allopatric combinations. \\
\hline dynamic polymorphism & $\begin{array}{l}\text { A selection regime that results in the maintenance of two or more alleles at a locus in a population but with } \\
\text { changing allele frequencies (often cyclic). }\end{array}$ \\
\hline selective sweep & $\begin{array}{l}\text { The process of an advantageous allele increasing to fixation under natural selection. Linked alleles may also go } \\
\text { to fixation by 'hitch-hiking'. }\end{array}$ \\
\hline $\begin{array}{l}\text { frequency-dependent } \\
\text { selection }\end{array}$ & $\begin{array}{l}\text { Dependence of relative genotypic fitnesses on genotype frequencies. Can be positive or negative. If negative, } \\
\text { that is, if the fitness of alleles at a locus declines with increasing frequency, a stable or dynamic polymorphism } \\
\text { may result. }\end{array}$ \\
\hline balancing selection & $\begin{array}{l}\text { Any form of selection acting to maintain alleles in a population, including frequency-dependent selection and } \\
\text { heterozygote advantage. }\end{array}$ \\
\hline positive selection & Spread of mutations owing to selection rather than drift. \\
\hline
\end{tabular}




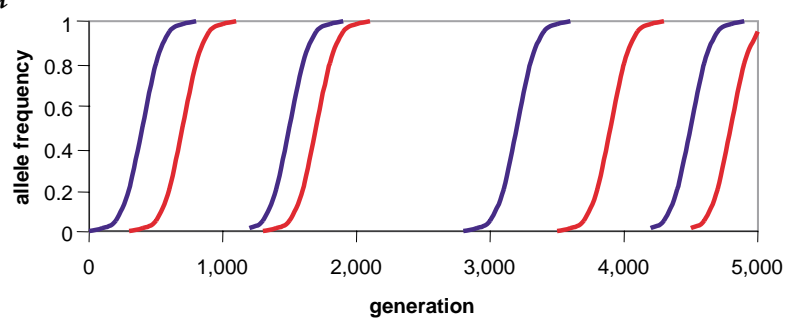

$b$

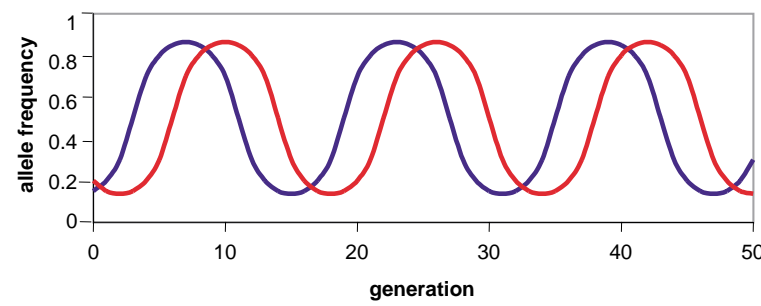

Fig. 2 Allele frequency changes driven by co-evolution. a, A series of selective sweeps by host (blue line) and pathogen (red) alleles derived by mutation. Selection is directional, that is, genetic change accumulates in both populations. At any given stage of the process there may be polymorphisms in either, both or neither of the two populations. In a large random-mating population, the time to fixation of a favorable variant is proportional to the reciprocal of the selection coefficient, but depends only logarithmically on the initial gene frequency. See Box 2, Equation 1. b, Dynamic polymorphisms in both host (blue) and pathogen (red) acting on existing genetic variation. Evolution is non-directional. At all stages of the process both populations are polymorphic. If pathogen genotypes track host genotypes closely, as illustrated, then sympatric host-pathogen combinations will generally be more compatible than allopatric (that is, genetically independent) ones: this is local adaptation. Because there is a lag between the cycles in host and pathogen, however, sympatric combinations will be less compatible than some allopatric combinations: this is local maladaptation. A large range of models predict, at least for certain parameter combinations, the kind of lagged limit cycles shown here. See Box 2, Equation 2.

("running as fast as you can to stay in the same place"). Unfortunately, both terms have been used to refer to both processes, even though their nature, time scale and population genetic consequences are quite distinct.

Conditions for co-evolution. Co-evolution requires (i) genetic variation (additive genetic variation for diploid species) in the relevant host and pathogen traits (ii) reciprocal effects of the traits involved on the fitnesses of the two populations and (iii) dependence of the outcome of the host-pathogen interaction on the combinations of genotypes involved (in multi-locus systems, this makes epistasis and linkage disequilibrium potentially important).

Evidence of these requirements is needed to demonstrate the potential for co-evolution. Demonstrating that co-evolution actually is occurring requires evidence of change (whether directional or non-directional) in both host and pathogen genotype frequencies in the field. In practice, such evidence is rarely available. This is not surprising: demonstrating any kind of ongoing selection is not straightforward ${ }^{23}$ and, even though it has long been believed that pathogens have an evolutionary effect on hosts $^{24}$, there are few unequivocal demonstrations of these effects $^{9,10}$. Demonstrating co-evolution presents the greater challenge of demonstrating reciprocal selection in both host and pathogen. It is likely to be most apparent where (i) a specialist pathogen exerts a strong selection pressure on its host as well as vice versa; (ii) the relevant host and pathogen traits have high heritability and a simple genetic basis; (iii) there are minimal other constraints on the relevant pathogen and host traits, including those due to the impact of selection imposed by other hosts or pathogens, respectively; and (iv) pathogen and host generation times are short. Because it is so difficult to observe the process of co-evolution, most studies have been concerned with demonstrating a pattern of variation in genotypes or phenotypes that is consistent with either ongoing or past co-evolution.

\section{Evidence for co-evolution}

Temporal patterns. There have been few long-term field studies of host-pathogen co-evolution. The classic example is the European rabbit-myxoma virus system in which, after the virus was introduced into a naïve rabbit population, phenotypic changes were observed in both pathogen and host components of virulence ${ }^{25}$ (Fig. $3 a$ ). The rapid, initial attenuation of the virus presumably represents adaptation to a novel host. But subsequent slower increases in both virus pathogenicity and the ability of the host to survive infection are consistent with co-evolution. Other evidence comes from a snail-trematode system that exhibits fluctuating frequencies of different host clones, such that recently rare clones are less susceptible to infection with contemporary pathogens, as expected with Red Queen co-evolution ${ }^{26,27}$.

Supporting evidence comes from work on model systems. Studies of foot-and-mouth virus in cell cultures have found increasing resistance of the cells to the virus, with accompanying

\section{Box $2 \cdot$ Equations}

- Equation 1. Time to fixation during a selective sweep is given by the expression ${ }^{92}$ :

$$
t=\frac{2}{s} \ln \left[\frac{p_{t}\left(1-p_{0}\right)}{p_{0}\left(1-p_{t}\right)}\right]
$$

where $t$ is time (in generations), $s$ is selection coefficient (assumed to be small), $p_{0}$ is initial allele frequency and $p_{t}$ is allele frequency at time $t$. A high proportion of selectively advantageous mutations are, however, lost by random sampling when they are still rare; the chance of survival is approximately twice the selective advantage of their carriers over the rest of the population ${ }^{92}$.

- Equation 2. An example of a model generating dynamics polymorphisms has the form ${ }^{16}$ :

$$
\frac{d M_{i}}{d t}=M_{i}\left[r_{i}(N)-\sum_{j=1}^{n} \beta_{i j} P_{i j}\right] ; \frac{d P_{i j}}{d t}=P_{i j}\left[\beta_{i j} M_{i}-\mu\right]
$$

where $M_{i}$ is the density of uninfected hosts with genotype $i ; P_{i j}$ is the density of hosts of genotype $i$ infected with pathogens of genotype $j_{i} N$ is the total host density; $r_{i}(N)$ is the density-dependent host population growth rate of genotype $i_{i} \beta_{i j}$ is per capita rate at which pathogens of genotype $j$ infect hosts of genotype $i$ (and is zero if a particular combination is not compatible); and $\mu$ is the per capita death rate of infected hosts. This is a matched genotype model for sexually reproducing hosts and pathogens. 


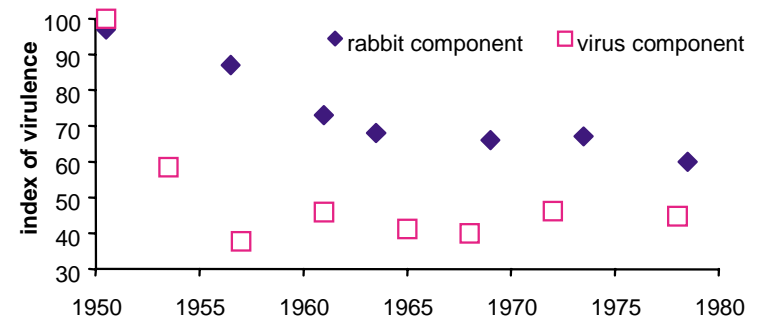

$b$

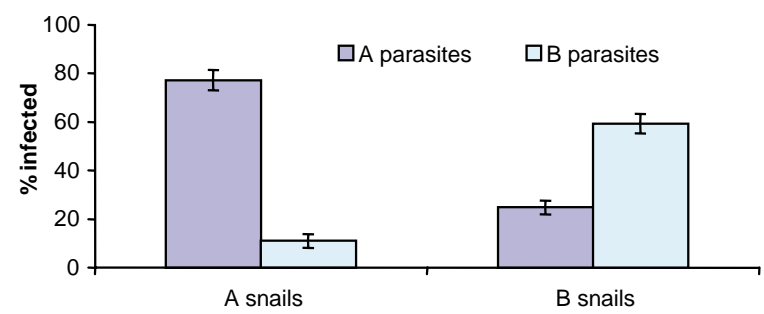

$\mathcal{C}$

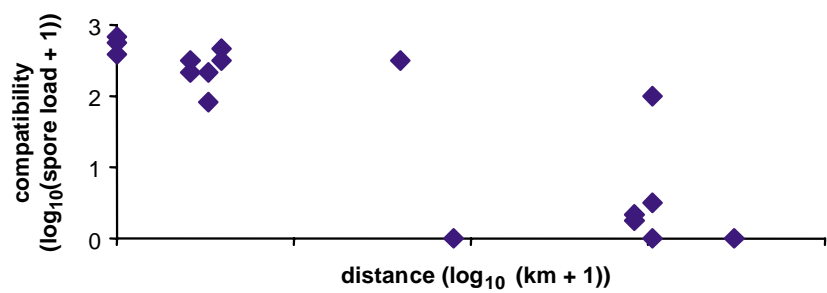

$d$

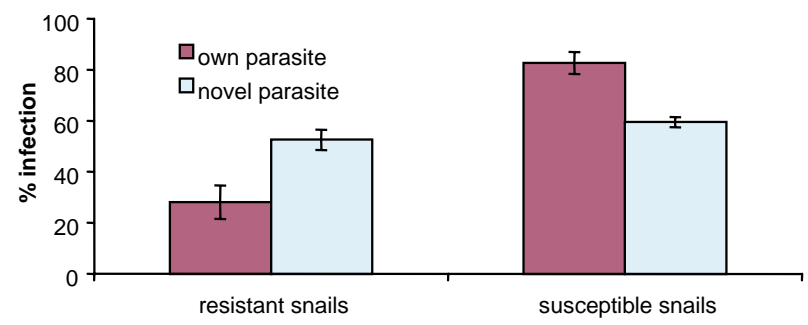

Fig. 3 Evidence for co-evolution. a, Temporal changes in pathogen and host components of virulence following the introduction of myxoma virus into the Australian populations of European rabbits. The virus component (as a weighted index of five case fatality grades among exposed reference laboratory rabbit lines) and the host component (as the percentage case fatality after exposure to reference laboratory virus isolates) are compared from 1950 onwards. Data are from ref. 25. $\boldsymbol{b}$, Local adaptation of the parasitic trematode Microphallus sp. to the freshwater snail Potamopygrus antipodarum. Percentage infection ( \pm 1 s.e.) resulting from exposures of all combinations of snails from two different lakes in New Zealand to equal doses of parasites from the same lakes. Sympatric combinations result in significantly higher percentage infection. Graph was redrawn from ref. 26. c, Decreasing compatibility with distance. Spore loads of the parasitic microsporidian Pleistophora intestinalis in Daphnia magna exposed to the same dose for combinations of different populations against distance between the host and parasite populations (log scale). More distant combinations result in significantly lower spore loads, that is, they are less compatible. Graph was redrawn from ref. 11. $d$, Strain-specific resistance and susceptibility of the freshwater snail Biomphalaria glabrata to the parasitic trematode Schistosoma mansoni. Percentage infection ( \pm 1 s.e.) of snail lines artificially selected for resistance and susceptibility when exposed to equal doses of different parasite strains. Resistance and susceptibility differed significantly from controls only for the parasite strain used for selection, not for a novel strain. Graph was redrawn from ref. 93.

phenotypic changes, and consequent selection for more pathogenic virus, with accompanying sequence variation in the VP1 gene ${ }^{28}$. Experimental studies of phage infections of Escherichia coli have shown that changes in a single gene, $\operatorname{LamB}$, can make the surface of the bacterium refractory to adsorption by a lytic phage, but that subsequent changes in a single phage gene can restore the ability of the phage to be adsorbed 29,30 . This leads to an arms race which can continue for a limited number of cycles ${ }^{6,7}$.

A practical difficulty in demonstrating co-evolution in action is that the time scales involved may be long, especially for hosts with long generation times. Unless the selection pressure imposed by pathogens is severe, selective sweeps can be slow (Box 2). Even if infection is fatal, selective sweeps by alleles conferring host resistance can still take hundreds of host generations if the pathogen has a long generation time of its own, such as scrapie in sheep or HIV in humans ${ }^{31,32}$.
Spatial patterns. Studies of spatial patterns thought to be consistent with co-evolution are rather more common. This approach implicitly assumes that spatial subpopulations are at different stages of a co-evolutionary process, so that spatial variation can be used to infer temporal variation ${ }^{22}$. Several theoretical models predict that sympatric host-pathogen combinations will generally be more compatible (that is, the pathogen is 'better' at infecting the host) than allopatric combinations-this is referred to as local adaptation-but that occasionally allopatric combinations will be most compatible-local maladaptation ${ }^{16,22}$ (Fig. $2 b$ ). But co-evolution need not always lead to local adaptation; for example, where virulence is low or migration rates are high ${ }^{22,33}$.

Local adaptation has been demonstrated in many host-pathogen systems (Table 1; Fig. $3 b$ ) with, as expected, local maladaptation being more rarely reported (Table 1). Compatibility has been shown not just to differ between sympatric and allopatric populations but to decrease with the distance between

\section{Table 1 Examples of spatial variation in pathogen-host compatibility11,16,22,26,94}

\section{Pathogen}

Local adaptation ${ }^{\mathrm{a}}$ :

Local maladaptation ${ }^{b}$ :
Microphallus sp. (a trematode) Schistosoma spp. (a trematode) Diplostomum phoxini (a trematode) Wuchereria bancrofti (a filarial worm) Pleistophora intestinalis (a microsporidian) Bluetongue virus Synchytrium decipiens (a fungus) Schistosoma mansoni (a trematode) Haemogregarine parasite

\section{Host}

Potamopygrus antipodarum (a snail) Bulinus globosus (a snail) European minnow Aedes polynesiensis (a mosquito) Daphnia magna (a crustacean) Culicoides variipenis (a midge) Amphicarpaea bracteata (an annual plant) Biomphalaria tenagophila (a snail) Gallotia galloti (a lizard) 
them (Fig. 3c). Local adaptation has also been found to reflect relatedness as well as sympatry. For example, a bacterium infecting the water flea Daphnia magna usually shows the highest compatibility with the host clones from which it was isolated, but is occasionally more compatible with novel host clones ${ }^{34}$.

These patterns are broadly consistent with the predictions of co-evolutionary theory, but they represent only indirect evidence and there may be alternative explanations for spatial variation in compatibility ${ }^{35-37}$. An important step is to establish that the observed patterns do indeed have a genetic basis ${ }^{26,38}$. Even then, however, it is possible that genetic divergence of local populations occurs independently of any interactions with other species. This is the probable explanation of the diversification of some pathogens within human subpopulations (for example, Helicobacter pylori, papillomaviruses and JC virus $\left.{ }^{39-41}\right)$, although local co-evolution could have occurred. Improved statistical methods for distinguishing genetic divergence from adaptive causes of correlated genetic change across populations ${ }^{42,43}$ can help interpret observed patterns. But the key point is that searching for co-evolution relies on the demonstration of reciprocal variation in pathogen and host phenotypes (and, ideally, genotypes too $)^{11,16,26}$. This constitutes much stronger evidence than spatial variation in either species alone ${ }^{38}$.

\section{Complications}

Genetic basis. One reason that co-evolution may be difficult to demonstrate in practice is that the genetic basis of host-pathogen interactions may be more complex than is represented by the simple gene-for-gene model. Resistance to pathogens is often a polygenic trait, especially in vertebrates ${ }^{44,45}$. Moreover, there may be a diversity of genetic mechanisms conferring increased host resistance; for example, there are many different ways in which humans are resistant to malaria ${ }^{46}$. And resistance may evolve not just by the appearance of new alleles at existing loci but through the accrual of new resistance mechanisms involving other loci ${ }^{17}$. Ultimately, it could be argued that many different host genes and, likewise, many different pathogen genes will have some direct or indirect influence on the outcome of the interaction, making it hard to identify the role of individual loci. Demonstrating reciprocal selection will be even harder in such situations.

Environmental as well as genetic factors will inevitably influence host-pathogen interactions and, particularly among vertebrates, there is the further complication of phenotypic plasticity ${ }^{47}$. The most obvious example is the adaptive immune response, which allows the same genetic machinery to be used to combat a vast number of different pathogens. And, in humans and perhaps other species, behavioral plasticity may also ameliorate the impact of pathogens. Phenotypic plasticity will reduce the importance of the host genotype-pathogen genotype interactions that drive co-evolution. The numerous examples of genetic variation in resistance to infection ${ }^{45}$ indicate, however, that environmental variation and phenotypic plasticity do not preclude evolutionary responses to pathogens.

\begin{tabular}{|c|c|}
\hline Receptor & Pathogens \\
\hline decay accelerating factor & echoviruses, Coxsackie viruses \\
\hline alpha-dystroglycan & $\begin{array}{l}\text { Lassa fever virus, lymphocytic } \\
\text { choriomeningitis virus }\end{array}$ \\
\hline sialic acid & $\begin{array}{l}\text { wide range including influenza } \\
\text { viruses and some reoviruses }\end{array}$ \\
\hline phosphate transporter molecule & $\begin{array}{l}\text { feline leukemia virus, } \\
\text { some retroviruses }\end{array}$ \\
\hline
\end{tabular}

Costs of resistance. An important complication is that, in the absence of infection, host resistance may carry a fitness cost, which may be apparent in such diverse ways as reduced survivorship $^{48}$, reduced fertility ${ }^{49}$, reduced competitive ability ${ }^{50}$ or increased susceptibility to non-communicable diseases ${ }^{51}$. A special case of a cost of resistance is heterozygote advantage, classically illustrated by the relationship between human sickle-cell anemia and susceptibility to malaria: the sickle-cell hemoglobin allele is homozygous lethal, and heterozygotes are resistant to malaria ${ }^{46}$. Heterozygote advantage may arise when the cost of resistance is recessive, as only the heterozygous effects of rare alleles are selected for in random-mating populations ${ }^{52}$.

Costs of resistance have several consequences. On the one hand, they can allow host polymorphisms to be maintained without any corresponding genetic variation in the pathogen and so without any co-evolution, although hosts might not invest in resistance at all if fitness costs are too high ${ }^{19}$. On the other hand, costs of resistance in the host coupled with costs of infectivity in the pathogen can drive co-evolution that would not otherwise occur ${ }^{19,53}$, though such costs are not a pre-requisite for co-evolution ${ }^{16,17}$.

Multiple pathogens, multiple hosts. Studies of co-evolution typically consider a single host species interacting with a single pathogen species. But, in reality, most host populations encounter a large number of different pathogen species ${ }^{54,55}$, and most pathogen species can infect more than one species of host-some can infect hosts from different taxonomic orders or even classes $^{54}$. Moreover, multiple infections with different strains of the same pathogen are common ${ }^{55,56}$. These multiple pathogen-multiple host interactions are not independent of one another, and this can be an important constraint on the potential for co-evolution.

There are several ways in which pathogen species interact within a host. At one level, pathogens may simply compete for host resources, and so directly influence each other's fitness. This may include sharing, and competing for, the same host receptors (Table 2). More subtly, infection with one species may enhance host susceptibility to another, a common effect known as facilitation, although the converse, inhibition, does occasionally occur (Table 3). Different pathogen species may not even be genetically independent: there is increasing evidence of the potential for genetic exchange between different prokaryote pathogens $s^{57,58}$.

There may also be interactions not just between different pathogen species within the host but between different genotypes or strains of the same species. These can have a number of effects. First, exposure to multiple pathogen strains can itself result in facilitation ${ }^{59,60}$, perhaps reflecting the advantages of antigenic diversity for escaping or overwhelming the immune response. Second, infections with multiple pathogen strains can also be differentially virulent ${ }^{56}$, possibly depending on how

Table 3 - Examples of interactions between pathogens ${ }^{\mathbf{9 7 - 1 0 1}}$
Facilitation
Positive associations occur between trypanosome and helminth
infections in mice.
Immunosuppressive effects of HIV or malaria can result in various
opportunistic infections.
Helminth infections affect the balance between the different
T cell types, Th1 and Th2, which in turn affects susceptibility to
other infections.
Echinostome infection predisposes snails to schistosome
infection.
Inhibition
Co-infection with hepatitis GBV-C can delay the progression of
HIV infection to AIDS.


closely the pathogens are related ${ }^{12,44}$, and can lead to complex selection pressures for higher or lower virulence ${ }^{61}$. Third, the underlying genetics may become more complicated; for example, different loci may be associated with resistance to different pathogen strains (Fig. $3 d$ ), and there may be epistasis between resistance genes, which reduces fitness costs associated with resistance to multiple pathogen strains, as has been reported for $E$. coli and phage ${ }^{62}$.

Having multiple hosts also affects the potential of pathogens to co-evolve with one host. On the one hand, for many pathogens, adaptation to a particular host population constrains its ability to infect other host populations ${ }^{44,61}$; this is supported by evidence from serial passage experiments ${ }^{63}$. There is even evidence that having a life cycle involving two or more host species constrains a pathogen's ability to co-evolve with either ${ }^{44,64}$. On the other hand, having multiple hosts may mean reduced selection pressure for a pathogen to co-evolve with any one host. If a pathogen can more easily evolve infectivity to a novel host than evade the resistance selected for in an existing host, this favors host switching. Although empirical support for this comes from brood parasites ${ }^{65}$ rather than pathogens, many viruses can exploit different host cell receptors, and this can be associated with host switching ${ }^{66}$, thereby demonstrating a potential for co-evolution involving multiple host species. But only certain pathogens may be capable of infecting multiple hosts; for example, viruses that use receptors conserved across different host taxa tend to have broader host ranges ${ }^{67}$. It is important to note that not all hosts exert any selection pressure on the pathogen at all. This particularly applies to 'accidental', 'dead-end' hosts (for example, humans for Toxoplasma gondii and Echinococcus granulosis), as these make no contribution to pathogen reproductive success ${ }^{1}$.

Clearly, a host population cannot evolve in response to selection pressure from one pathogen population without affecting the selection pressures imposed by other pathogens, and a pathogen population cannot evolve in response to selection pressure from one host population without affecting the selection pressures imposed by other host populations. The conceptual models of co-evolution in single pathogen-single host systems that currently dominate the literature may not always be appropriate in a multiple pathogen-multiple host world.
Molecular basis of co-evolution

Molecular interactions. The evidence for co-evolution discussed so far is phenomenological; an alternative approach is to look for evidence for co-evolution at the biochemical level. The molecular biology underlying host-pathogen co-evolution is, in general, only poorly understood, but there is a huge literature on molecule-for-molecule interactions between hosts and pathogens, and these are obvious candidates for coevolution (Table 4). A potentially important category is virus receptors ${ }^{68}$, many of which are proteins involved in host immune responses, itself raising interesting questions about the co-evolution of the immune system and its targets. Functional mimicry of host molecules (for example, Salmonella SptP mimicking host GAP enzymes or, commonly, mimicry to evade the immune response ${ }^{69}$ ) also raises the possibility of molecule-for-molecule co-evolution, although there is not yet evidence that hosts do, or can, respond by evading the mimics. To date, few recognized host-pathogen molecular interactions have been examined with co-evolution in mind, but there are several instances in which studies of underlying patterns of genetic variation are providing indications of the potential, at least, for molecular co-evolution. These include studies of molecular polymorphisms, gene genealogies and positive selection.

Molecular polymorphisms. Functional polymorphisms are often found in pathogen genes coding for antigens, that is, molecules that interact with the immune system, but there are also examples involving other kinds of interaction with the host, such as with host cell receptors (Table 4). Many host cell receptors for viruses are themselves polymorphic (Table 4), although this does not necessarily indicate a potential for co-evolution, as most of these polymorphisms are to do with inherited disorders and are not known to affect pathogen infections. Some host genes, however, are involved with both. An allele of SLC11A1, a gene involved in macrophage activation, increases resistance to tuberculosis but is also associated with autoimmune disease ${ }^{51}$, illustrating that constraints on co-evolution owing to costs of resistance can be identified at the molecular level. Notably, this implies that interactions with pathogens can also be important in the evolutionary origins of susceptibility to non-communicable as well as infectious diseases ${ }^{1}$.

These examples concern polymorphisms in only the host or only the pathogen, however; there are few instances in which reciprocal molecular polymorphisms have been identified. One candidate is the instance of human MHC and malaria CS genes in west African populations. Different MHC alleles have different abilities to bind to a variable region of the CS protein, and analyses of the co-distributions of pathogen and host genotypes are consistent with selection pressures exerted by each on the other ${ }^{70}$. There may also be potential for molecular co-evolution between humans and HIV. Susceptibility to HIV/AIDS varies across alleles encoding the CCR5 receptor. The resis- 
Fig. 4 Illustration of co-evolution between two host populations (blue rectangles) and four pathogen populations (red circles). Pathogens use specific host receptors (small triangles, squares and circles, matched by shape and color). Two pathogens compete for the same host receptor; otherwise, the pathogens use three different host receptors and 'cooperate' by facilitating the overwhelming of the host immune response. The result is a complex web of positive (arrows) or negative (circles), strong (solid lines, indicating good match to host receptor) or weak (broken lines, indicating a poor match to host receptor) interactions between the six populations. Selection for different receptor expression by the host may alter susceptibility to more than one pathogen population; selection for different receptor usage by the pathogen may alter infectivity to more than one host population.

tant alleles are deletion mutants that probably arose several hundred years ago and appear to have been subject to strong selection $^{71}$, possibly caused by another pathogen such as Yersinia pestis ${ }^{66,72}$. In addition, variation in the HIV env gene is associated with variation in infectivity to different CCR5 variants ${ }^{73,74}$. There is also some evidence for a potentially co-evolutionary interaction between HIV-1 and human CTL responses $^{75}$, although the CTL response also varies independently of the pathogen ${ }^{76-78}$.

Gene genealogies. Phylogenetic methods can be used to trace the evolutionary histories of alleles implicated in host-pathogen co-evolution ${ }^{71,79}$. Alleles differing at few sites have probably evolved relatively recently, and may represent transient polymorphisms (Fig. 2a). In contrast, alleles with large sequence differences probably have ancient origins and have been maintained by selection, possibly imposed by co-evolution. One possible example is provided by plant $R$-gene alleles ${ }^{79}$, although enhanced variability due to gene conversion between members of $R$-gene families may also explain the observed patterns. Another is the mammalian MHC: some MHC alleles appear to have been maintained for tens of millions of years ${ }^{80}$. MHC alleles are implicated in host resistance polymorphisms for a wide variety of pathogens, and are used as receptors by several different viruses, so there is potential for dynamic polymorphisms $^{81,82}$. It is unclear, however, whether MHC alleles are maintained by frequency-dependent selection or by heterozygote advantage $^{9}$, and to what extent resistance to pathogens is in fact the main selection pressure. Plant $R$-genes and mammalian MHC do suggest, however, that host genetic diversity can be maintained for extremely long time periods, consistent with powerful balancing selection, possibly involving interactions with many different pathogens ${ }^{79,81}$.

Positive selection. An alternative approach to identifying potential molecular co-evolution is to look for evidence of positive selection in genes involved in host-pathogen interactions, the argument being that, if co-evolution accelerates the accumulation of genetic variation, then positive selection should be more likely where co-evolution is acting ${ }^{9,79}$, although this is not universally accepted ${ }^{83}$. One way to look for positive selection involves comparing the ratio of non-synonymous (amino acid-changing) to synonymous (silent) nucleotide substitutions ${ }^{79,83}$, although interpreting the data is not always straightforward ${ }^{84,85}$. But positive selection has been reported for a wide variety of host genes involved in resistance to pathogens and also for several pathogen genes (Table 4). A difficulty is that most genetic variability in pathogens of vertebrates probably reflects selection of antigens to evade host immune responses, whose diversity is generated outside the germ line, and so does not involve true co-evolution. A recent study of influenza A hemagglutinin did, however, report evidence of positive selection in the receptor binding site as well as in known epitopes ${ }^{86}$.

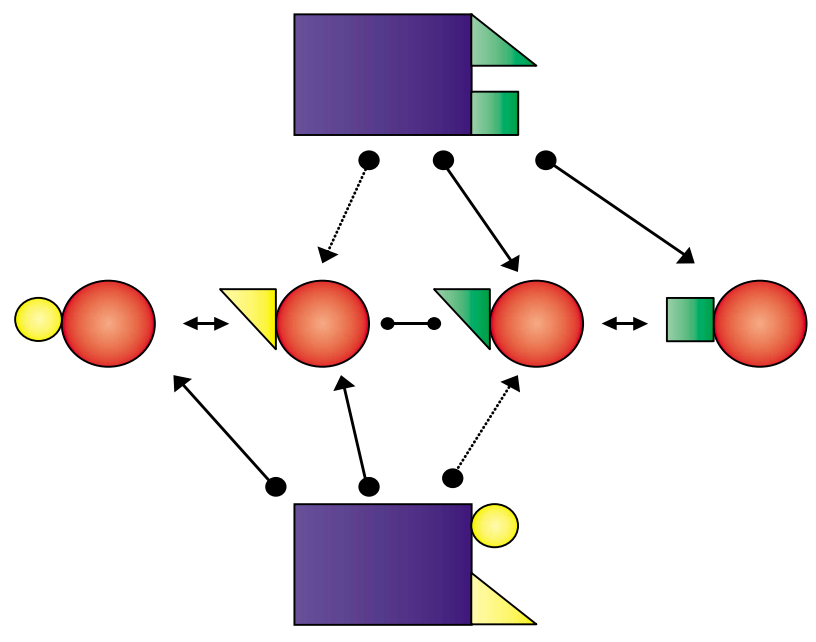

Future of co-evolution

There is evidence consistent with co-evolution, but most of it is indirect and concerns plant, invertebrate and bacteria hosts. For vertebrate hosts, especially humans, evidence for co-evolution is sparser, but there are many indications of potential for co-evolution and, therefore, its significance is an open and important question. Part of the problem is that there are many reasons why demonstrating co-evolution involving vertebrate hosts may be particularly difficult: lack of understanding of either the genetics or molecular basis of the host-pathogen interaction; involvement of polygenic traits; fitness constraints on these traits, including constraints imposed by simultaneous interactions with multiple hosts or multiple pathogens; phenotypic plasticity; and the long time scales involved.

One way of addressing these issues is to develop new, more powerful methods for searching for evidence of co-evolution, beyond the traditional phenomenological approaches favored by evolutionary biologists. We suggest that a good starting point is to identify reciprocal polymorphisms in genes involved in the host-pathogen interaction at the molecular level-these genes are obvious candidates for co-evolution. This approach will require data on nucleotide variation in both pathogen and host populations ${ }^{87,88}$, accompanied by use of the battery of tests for selection that are now available to sequence data ${ }^{23,89}$. A complementary approach would be to look for variation in gene expression during infections of different host genotypes by different pathogen genotypes. The key is to seek reciprocal variation by studying host and pathogen in parallel.

There is already abundant evidence for, and huge interest in, genetic variation in host susceptibility to infectious diseases. Correctly interpreting this variation requires an understanding of the potentially dynamic nature of the genetics of the host-pathogen interaction. If co-evolution is involved, then the susceptibility of a particular host genotype to an infectious disease depends on the genotypes of the pathogens to which it is exposed: an allele conferring protection at one place and time may have the opposite effect at another.

We anticipate that further evidence will be found for co-evolution, as is hinted at by the ongoing studies of human interactions with malaria and HIV discussed earlier. Moreover, we suspect that the subset of host genes involved in co-evolutionary interactions will turn out to be of particular biomedical importance, as the examples of mammalian MHC and plant $R$-genes imply. This is because they probably determine susceptibility and pathogenicity, and should provide insights not only into the ways that host and pathogen adapt and counter-adapt, but, perhaps more 
importantly, into the constraints which prevent either from 'winning' the evolutionary battle outright. Moreover, understanding the responses of pathogens to evolved changes in host characteristics may provide a good model of their all-too-apparent potential to respond to other kinds of change, such as the use of new drugs or vaccines to combat disease $\mathrm{e}^{63,88,90}$.

Co-evolution will also have epidemiological implications, particularly in the context of emerging and re-emerging diseases ${ }^{91}$. If co-evolution imposes constraints on susceptibility and pathogenicity, those constraints may no longer hold when new host-pathogen associations emerge or ancient associations are disrupted, affecting both the magnitude and severity of disease outbreaks. This applies not only when host populations are exposed to novel pathogens but also when they are exposed to different populations of existing pathogens. There may also be indirect effects of changes in the range of pathogens to which a host population is exposed through altering the selection pressures on existing pathogens (Fig. 4). Altering host genetics, especially by selective breeding for resistance to a particular pathogen, could also affect selection pressures on other pathogens. The deliberate introduction of novel pathogens as a means of biological control will probably be followed by changes in the pathogen and, quite possibly, the target host population ${ }^{25}$.

Recent interest in 'evolutionary medicine' represents a potential starting point for developing these ideas ${ }^{1}$. But most evolutionary biologists tend to work at the population level and pay little attention to the molecular basis of infection and disease, whereas most biomedical scientists tend to work at the molecular and cellular level and pay little attention to population processes. A fuller understanding of the biological and biomedical implications of co-evolution demands closer links between these disciplines than exists at present, but will be greatly to the benefit of both.

\section{Acknowledgments}

We thank A. Read, L. Taylor, D. Haydon, M. Charleston, R. Phillips, C. Davies, P. Goulder, C. Lively and E. Baranowski for comments and discussion and gratefully acknowledge support from the Wellcome Trust, the Leverhulme Trust, the Royal Society, Ministerio de Ciencia y Tecnologia and Fundacion R Areces.

\section{Competing interests statement}

The authors declare that they have no competing financial interests.

Received 17 June; accepted 9 September 2002.

1. Stearns, S.C. Evolution in Health \& Disease (Oxford University Press, Oxford, 1999).

2. Rainey, P.B., Buckling, A., Kassen, R. \& Travisano, M. The emergence and maintenance of diversity: insights from bacterial populations. Trends Microbiol. 15, 243-247 (2000)

3. Thompson, J.N. \& Burdon, J.J. Gene-for-gene coevolution between plants and parasites. Nature 360, 121-125 (1992).

4. Thompson, J.N. The Coevolutionary Process (University of Chicago Press, Chicago, 1994).

5. Flor, H.H. The complementary genic systems in flax and flax rust. Adv. Genet. 8 29-54 (1956).

6. Chao, L., Levin, B.R. \& Stewart, F.M. A complex community in a simple habitat: an experimental study with bacteria and phage. Ecology 58, 369-378 (1977).

7. Lenski, R.E. \& Levin, B.R. Constraints on the coevolution of bacteria and virulent phage: a model, some experiments, and predictions for natural communities. Am. Nat. 125, 585-602 (1985).

8. Kniskern, J. \& Rausher, M.D. Two modes of host-enemy evolution. Popul. Ecol. 43, 3-14 (2001).

9. Little, T.J. The evolutionary significance of parasitism: do parasite-driven dynamics occur ex silico? J. Evol. Biol. 15, 1-9 (2002).

10. Satta, Y., Ohuigin, C., Takahata, N. \& Klein, J. Intensity of natural selection at the major histocompatibility complex loci. Proc. Natl Acad. Sci. USA 91, 7184-7188 (1994).

11. Ebert, D. Virulence and local adaptation of a new horizontally transmitted parasite. Science 265, 1084-1086 (1994).

12. Frank, S.A. Models of parasite virulence. Q. Rev. Biol. 71, 37-78 (1996)

13. Levin, B.R. The evolution and maintenance of virulence in microparasites. Emerg. Infect. Dis. 2, 93-102 (1996).

14. Mode, C.J. A mathematical model for the coevolution of obligate parasites and their hosts. Evolution 12, 138-165 (1958).
15. Hamilton, W.D., Axelrod, R. \& Tanese, R. Sexual reproduction as an adaptation to resist parasites (a review). Proc. Natl Acad. Sci. USA 87, 3566-3573 (1990).

6. Morand, S., Manning, S.D. \& Woolhouse, M.E.J. Parasite-host coevolution and geographic patterns of parasite infectivity and host susceptibility. Proc. $R$. Soc. Lond. B Biol. Sci. 263, 119-128 (1996).

17. Lively, C.M. \& Apanius, V. Genetic diversity in host-parasite interactions. in Ecology of Infectious Diseases in Natural Populations (eds Grenfell, B.T. \& Dobson, A.P.) 421-449 (Cambridge University Press, Cambridge, 1995).

18. Anderson, R.M. \& May, R.M. Infectious Diseases of Humans: Dynamics and Control (Oxford University Press, Oxford, 1991).

19. Sasaki, A. \& Godfray, H.C.J. A model for the coevolution of resistance and virulence in coupled host-parasitoid interactions. Proc. R. Soc. Lond. B Biol. Sci. 266, 455-463 (1999).

20. Gandon, S. \& Michalakis, Y. Evolution of parasite virulence against qualitative or quantitative resistance. Proc. R. Soc. Lond. B Biol. Sci. 267, 985-990 (2000).

21. Sasaki, A. Host-parasite coevolution in a multilocus gene-for-gene system. Proc. R. Soc. Lond. B Biol. Sci. 267, 2183-2188 (2000).

22. Gandon, S. Local adaptation and the geometry of host-parasite coevolution. Ecol. Lett. 5, 246-256 (2002).

23. Kreitman, M. Methods to detect selection in populations with applications to humans. Ann. Rev. Genomics Hum. Genet. 1, 539-559 (2000).

24. Haldane, J.B.S. Disease and evolution. Ric. Sci. 19,68-76 (1949).

25. Fenner, F. \& Fantini, B. Biological Control of Vertebrate Pests (CABI Publishing, Wallingford, 1999).

26. Lively, C.M. \& Dybdahl, M.F. Parasite adaptation to locally common host genotypes. Nature 405, 679-681 (2000).

27. Dybdahl, M.F. \& Lively, C.M. Host-parasite coevolution: evidence for rare advantage and time-lagged selection in a natural population. Evolution 52. 1057-1066 (1998)

28. de la Torre, J.C. et al. Coevolution of cells and viruses in a persistent infection of foot-and-mouth disease virus in cell culture. J. Virol. 62, 2050-2058 (1988).

29. Braun-Breton, C. \& Hofnung, M. In vivo and in vitro functional alterations of the bacteriophage lambda receptor in lamB missense mutants of Escherichia coli K12. J. Bacteriol. 148, 845-852 (1981).

30. Hofnung, M., Jezierska, A. \& Braun-Breton, C. LamB mutations in E. coli K12: growth of lambda host range mutants and effect of nonsense suppressors. $\mathrm{Mol}$. Gen. Genet. 145, 207-213 (1976).

31. Woolhouse, M.E.J. et al. A centuries-long epidemic of scrapie in British sheep? Trends Microbiol. 6, 67-70 (2001)

32. Levin, B.R., Bull, J.J. \& Stewart, F.M. The intrinsic rate of increase of HIVIAIDS: epidemiological and evolutionary implications. Math. Biosci. 132, 69-96 (1996).

33. Lively, C.M. Migration, virulence and the geographic mosaic of adaptation by parasites. Am. Nat. 153, S34-\$47 (1999).

34. Carius, H.J., Little, T.J. \& Ebert, D. Genetic variation in a host-parasite association: potential for coevolution and frequency-dependent selection. Evolution 55, 1136-1145 (2001).

35. Thompson, J.N. Specific hypotheses on the geographical mosaic of coevolution. Am. Nat. 153, S1-S14 (1999).

36. Nuismer, S. L., Thompson, J.N. \& Gomulkiewicz, R. Gene flow and geographically structured coevolution. Proc. R. Soc. Lond. B Biol. Sci. 266, 605-609 (1998).

37. Kraaijeveld, A.R. \& Godfray, H.C.J. Geographic patterns in the evolution of resistance and virulence in Drosophila and its parasitoids. Am. Nat. 153, S61-S74 (1999).

38. Woolhouse, M.E.J. \& Webster, J.P. In search of the Red Queen. Parasitol. Today, $16,506-508(2000)$.

39. Covacci, A., Telford, J.L., Del Giudice, G., Parsonnet, J. \& Rappuoli, R. Helicobacter pylori virulence and genetic geography. Science 284, 1328-1333 (1999).

40. Ong, C.K. et al. Evolution of human papillomavirus type 18: an ancient phylogenetic root in Africa and intratype diversity reflect coevolution with human ethnic groups. J. Virol. 67, 6424-6423 (1993).

41. Agostini, H.T., Yanagihara, R., Davis, V., Ryschkewitsch, C.F. \& Stoner, G.L. Asian genotypes of $\mathrm{JC}$ virus in native Americans and in a Pacific island population: markers of viral evolution and human migration. Proc. Natl Acad. Sci. USA 94, 14542-14546 (1997).

42. Harvey, P.H. \& Pagel, M.D. The Comparative Method in Evolutionary Biology (Oxford University Press, Oxford, 1991).

43. Huelsenbeck, J.P., Rannala, B. \& Masly, J.P. Accommodating phylogenetic uncertainty in evolutionary studies. Science 288, 2349-2350 (2000).

44. Webster, J.P. \& Davies, C.M. Coevolution and compatibility in the snailschistosome system. Parasitology 123, S42-S56 (2001).

45. Wakelin, D. \& Blackwell, J.M. (eds). Genetics of Resistance to Bacterial and Parasitic Infection (Taylor \& Francis, London, 1988).

46. Hill, A.V.S., Jepson, A., Plebanski, M. \& Gilbert, S.C. Genetic analysis of host-parasite coevolution in human malaria. Philos. Trans. R. Soc. Lond. B Biol. Sci. 352, 1317-1325 (1997).

47. Agrawal, A.A. Phenotypic plasticity in the interactions and evolution of species. Science 294, 321-326 (2001).

48. Yan, G., Severson, D.W. \& Christensen, B.M. Costs and benefits of mosquito refractoriness to malaria parasites: implications for genetic variability of mosquitoes and genetic control of malaria. Evolution 51, 441-450 (1997).

49. Webster, J.P. \& Woolhouse, M.E.J. Cost of resistance: relationship between reduced fertility and increased resistance in a Schistosoma host-parasite system. Proc. R. Soc. Lond. B Biol. Sci. 266, 391-396 (1999).

50. Kraaijeveld, A.R. \& Godfray, H.C.J. Trade-off between parasitoid resistance and larval competitor ability in Drosophila melanogaster. Nature 389, 278-280 (1997).

51. Searle, S. \& Blackwell, J.M. Evidence for a functional-repeat polymorphism in the promoter of human NRAMP1 gene that correlates with autoimmune versus infectious disease susceptibility. J. Med. Gen. 36, 295-299 (1999).

52. Falconer, D.S. \& Mackay, T.F.C. An Introduction to Quantitative Genetics, 4th Edition (Longman, London, 1996).

53. Agrawal, A. \& Lively, C.M. Infection genetics: gene-for-gene versus matching allele models, and all points in between. Evol. Ecol. Res. 4, 1-12 (2002).

54. Taylor, L.H., Latham, S.M. \& Woolhouse, M.E.J. Risk factors for human disease emergence. Philos. Trans. R. Soc. Lond. B Biol. Sci. 356, 983-990 (2001).

55. Webster, J.P. \& Macdonald, D.W. Parasites of wild brown-rats (Rattus norvegicus) on UK farms. Parasitology 111, 247-255 (1995). 
56. Read, A.F. \& Taylor, L.R. The ecology of genetically diverse infections. Science 292, 1099-1102 (2001)

57. Ochman, H. \& Moran, N.A. Genes lost and genes found: evolution of bacterial pathogenesis and symbiosis. Science 292, 1096-1098 (2001).

58. Fraser, C.M., Eisen, J.A. \& Salzberg, S.L. Microbial gene sequencing. Nature 406 799-803 (2000).

59. Lord, C.C. et al. Aggregation and distribution of strains in microparasites. Philos. Trans. R. Soc. Lond. B Biol. Sci. 354, 799-807 (1999).

60. Basanez, M.G. et al. Density-dependent processes in the transmission of human onchocerciasis-relationship between the numbers of microfilariae ingested and successful larval development in the simuliid vector. Parasitology 110 409-427 (1995).

61. Woolhouse, M.E.J., Taylor, L.H. \& Haydon, D.T. Population biology of multi-host pathogens. Science 292, 1109-1112 (2001).

62. Bohannan, B.J.M., Travisano, M. \& Lenski, R.E. Epistatic interactions can lowe the cost of resistance to multiple consumers. Evolution 53, 292-295 (1999).

63. Ebert, D. Experimental evolution of parasites. Science 282, 1432-1435 (1998).

64. Weaver, S.C., Brault, A.C., Kang, W.L. \& Holland J.J. Genetic and fitness changes accompanying adaptation of an arbovirus to vertebrate and invertebrate cells. $J$. Virol. 73, 4316-4326 (1999).

65. Davies, N.B. \& de L. Brooke, M. An experimental study of co-evolution between the cuckoo, Cuculus canorus, and its hosts. II Host egg markings, chick discrimination and general discussion. J. Anim. Ecol. 58, 225-236 (1989).

66. Schneider-Schaulies, J. Cellular receptors for viruses: links to tropism and pathogenesis. J. Gen. Virol. 81, 1413-1429 (2000).

67. Woolhouse, M.E.J. Population biology of emerging and re-emerging pathogens. Trends Microbiol. 10, S3-S7 (2002)

68. Baranowski, E., Ruiz-Jarabo, C.M. \& Domingo, E. Evolution of cell recognition by viruses. Science 292, 1102-1105 (2001).

69. Stebbins, C.E. \& Galan, J.E. Structural mimicry in bacterial virulence. Nature $\mathbf{4 1 2}$ 701-705 (2001)

70. Gilbert, S.C. et al. Association of malaria parasite population structure, HLA, and immunological antagonism. Science 279, 1173-1177 (1998).

71. Slatkin, M. \& Rannala, B. Estimating allele age. Ann. Rev. Genomics Hum. Genet. 1, 225-249 (2000).

72. Altschuler, E.L. Plague as HIV vaccine adjuvant. Med. Hypotheses 54, 1003-1004 (2000).

73. Schliekelman, P., Garner, C. \& Slatkin, M. Natural selection and resistance to HIV. Nature 411, 545-546 (2001).

74. Berger, E.A., Murphy, P.M. \& Farber, J.M. Chemokine receptors as HIV-1 coreceptors: roles in viral entry, tropism and disease. Ann. Rev. Immunol. 17, 657-700 (1999).

75. Moore, C.B. et al. Evidence of HIV-1 adaptation to HLA-restricted immun responses at a population level. Science 296, 1439-1443 (2002).

76. Day, C.L. et al. Relative dominance of epitope-specific cytotoxic T-lymphocyte responses in human immunodeficiency virus type 1-infected persons with shared HLA alleles. J. Virol. 75, 6279-6291 (2001).

77. Goulder, P.J.R. et al. Patterns of immunodominance in HIV-1-specific cytotoxic lymphocyte responses in two human histocompatibility leukocyte antigen (HLA)-identical siblings with HLA-A*0201 are influenced by epitope mutation. $J$. Exp. Med. 185, 1423-1433 (1997).

78. Altfeld, M. et al. Cellular immune responses and viral diversity in individuals treated during acute and early HIV-1 infection. J. Exp. Med. 193, 169-180 (2001).

79. Bergelson, J., Kreitman, M., Stahl, E.A. \& Tian, D.C. Evolutionary dynamics of plant $R$-genes. Science 292, 2281-2285 (2001).

80. Klein, J., Satta, Y., Ohuigin, C. \& Takahata, N. The molecular descent of the major histocompatibility complex. Ann. Rev. Immunol. 11, 269-295 (1993)

81. Vogel, T.U. et al. Major histocompatibility complex class I genes in primates: coevolution with pathogens. Immunol. Rev. 167, 327-337 (1999).

82. Apanius, V., Penn, D., Slev, P.R., Ruff, L.R. \& Potts, W.K. The nature of selection on the major histocompatibility complex. Crit. Rev. Immunol. 17, 179-224 (1997).

83. Yang, Z. \& Bielawski, J.P. Statistical methods for detecting molecular adaptation Trends Ecol. Evol. 15, 496-503 (2000)

84. Vartanian, J.P., Henry, M. \& Wain-Hobson, S. Simulating pseudogene evolution in vitro: determining the true number of mutations in a lineage Proc. Natl Acad. Sci. USA 98, 13172-13176 (2001).

85. Domingo, E., Webster, R.G. \& Holland, J. Origin and Evolution of Viruses (Academic Press, San Diego, 1999)

86. Bush, R.M. et al. Predicting the evolution of human influenza A. Science 286 , 1921-1925 (1999).

87. International SNP Map Working Group. A map of human genome sequence variation containing 1.42 million single-nucleotide polymorphisms. Nature $\mathbf{4 0 9}$, 928-933 (2001).

88. Domingo, E., Biebricher, C., Eigen, M. \& Holland, J.J. Quasispecies and RNA Virus Evolution: Principles and Consequences (Landes Bioscience, Landes, 2001).

89. Charlesworth, D., Charlesworth, B. \& McVean, G. Genome sequences and molecular evolution, a two-way interaction. Trends Ecol. Evol. 16, 235-242 (2001).

90. Gandon, S., Mackinnon, M.J., Nee, S. \& Read, A.F. Imperfect vaccines and the evolution of pathogen virulence. Nature 414, 751-756 (2001)

91. Woolhouse, M.E.J. \& Dye, C. (eds) Population biology of emerging and reemerging pathogens. Philos. Trans. R. Soc. Lond. B Biol. Sci. 356, 979-1106 (2001).

92. Crow, J.F. \& Kimura, M. An Introduction to Population Genetics Theory (Harper and Row, New York, 1970).

93. Webster, J.P. \& Woolhouse, M.E.J. Heritability and strain specificity in compatibility between snail intermediate hosts and their parasitic schistosomes. Evolution 52, 1627-1634 (1998).

94. Oppliger, A., Vernet, R. \& Baez, M. Parasite local maladaptation in the Canarian lizard Gallotia galloti (Reptilia: Lacertidae) parasitized by haemogregarian blood parasite. J. Evol. Biol. 12, 951-955 (1999).

95. Cao, W. et al. Identification of $\alpha$-dystroglycan as a receptor for lymphocytic choriomeningitis virus and Lassa fever virus. Science 282, 2079-1081 (1998).

96. Overbaugh, J. \& Bangham, C.R.M. Selection forces and constraints on retroviral sequence variation. Science 292, 1106-1109 (2001).

97. Sorci, G., Moller, A.P. \& Boulinier, T. Genetics of host-parasite interactions. Trends Ecol. Evol. 12, 196-200 (1997).

98. Helmby, H., Kullberg, M. \& Troye-Blomberg, M. Altered immune responses in mice with concomitant Schistosoma mansoni and Plasmodium chabaudi infections. Infect. Immun. 66, 5167-5174 (1998).

99. Allen, J.E. \& Maizels, R.M. Th1-Th2: reliable paradigm or dangerous dogma? Immunol. Today 18, 387-392 (1997).

100. Sullivan, J.T. et al. Schistosoma mansoni, NIH-SM-PR-2 strain, in non-susceptible Biomphalaria glabrata-protection by Echinostoma paraensei. Int. J. Parasitol. 11, 481-484 (1981).

101. Stosor, V. \& Wolinsky, S. GB virus C and mortality from HIV infection. N. Engl. J. Med. 345, 761-762 (2001).

102. Donnenberg, M.S. Pathogenic strategies of enteric bacteria. Nature 406, 768-774 (2000).

103. Pellizzari, A., Pang, H. \& Lingwood, C.A. Binding of verocytotoxin 1 to its receptor is influenced by differences in receptor fatty acid content. Biochemistry 31, 1363-1370 (1992).

104. McFadden, G. Getting to know you-viruses meet CD40 ligand. Nature Med. 1, 408-409 (1995).

105. Faure, S. et al. Rapid progression to AIDS in HIV+ individuals with a structural variant of the chemokine receptor CX(3)CR1. Science 287, 2274-2277 (2000). 